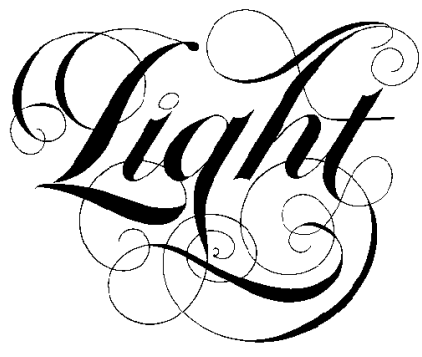

\title{
Intensity Profile in a Distributed Feedback Fibre Laser Characterised by a Green Fluorescence Scanning Technique
}

\author{
W.H.Loh, B.N.Samson and J.P.de Sandro
}

\begin{abstract}
We report on an experimental technique for investigating the intensity profile in distributed feedback fibre lasers. By scanning along the laser length and monitoring the side green fluorescence, the intensity distribution in the laser can be inferred. With this approach, we experimentally verify the sharply peaked intensity distribution predicted for $\lambda / 4$ phase-shifted distributed feedback lasers.
\end{abstract}

The nonuniform intensity profiles inherent in distributed feedback (DFB) semiconductor lasers are of considerable concern, as they can lead to single mode instability through spatial hole burning effects [1-3]. For the well-known discrete $\lambda / 4$-phase shifted DFB laser [4], the nonuniformity of the field distribution is particularly severe in strong gratings. Theoretical analyses of this structure have shown that the lasing distribution is sharply peaked in the vicinity of the phase shift, prompting considerable effort towards reducing this peak in the intensity profile, e.g. through chirped DFB structures [3]. However, apart from a limited number of reports confirming the presence of spatial hole burning in DFB lasers through spatially resolved spontaneous emission measurements $[5,6]$, there has been, to our knowledge, no experimental verification of the intensity distribution according to theory.

The lack of experimental verification is quite understandable. With the small size of semiconductor DFB lasers, achieving the spatial resolution with sufficient signal-to-noise ratio is not easy. Worse, large refractive index changes accompany the spatial hole burning, effectively chirping the grating, thus distorting the DFB structure and hence the intensity distribution. With the recent development of DFB $\mathrm{Er}^{3+}$-fibre lasers [7-9], however, the prospects for direct confirmation of the theoretical distribution profiles are promising. The gain-induced refractive index changes are typically small, $\sim 10^{-7}$ for moderately doped fibres [10], thus distortions to the grating due to spatial hole burning is expected to be minimal. And with centimeter long lasers, achieving the spatial resolution to observe any sharp variations in the distribution is clearly easier. In this work, we report on experimental investigations with the view towards mapping out the intensity profile in a DFB Er ${ }^{3+}$-fibre laser, by monitoring the green fluorescence from pump excited state absorption.

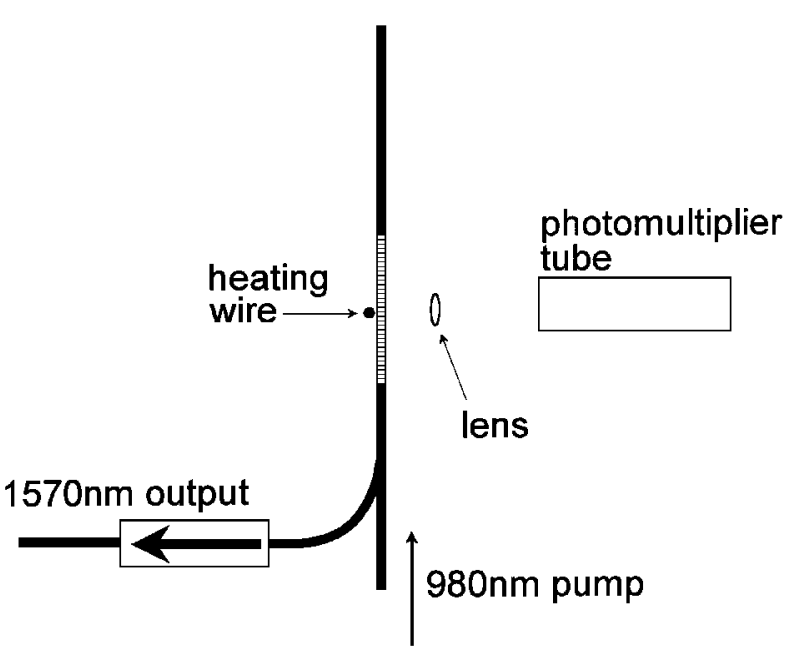

The experimental technique is simple, consisting only of scanning the length of the DFB $\mathrm{Er}^{3+}$-fibre laser and monitoring the Fig. 1 Experimental configuration. green fluorescence (Fig. 1). One advantage worth noting is that while the DFB laser is operating in the infrared $(1.55 \mu \mathrm{m})$ regime, the low-level fluorescence measurements are in the visible. Thus, 
conventional photomultipliers can be used, providing the sensitivity for picking up the visible fluorescence while remaining insensitive to artifacts from scattered lasing (infrared) emission (we note, however, that Rayleigh sidescatter of injected infrared radiation can be used to determine optical resonances in fibre gratings) [11]. For this experiment, a $5 \mathrm{~cm}$ long DFB fibre laser was used, made by exposing the $\mathrm{Er}^{3+}$-doped germanosilicate fibre to uv radiation against a uniform phase mask. The erbium fibre was moderately doped, with a small-signal pump absorption of $4 \mathrm{~dB} / \mathrm{m}$. The grating strength is estimated to be $\mathrm{L} 6.9\left(\mathrm{n} \sim 1 \mathrm{O}^{-4}\right)$, from reflectivity measurements on a short grating written under identical conditions. A phase shift in the grating is induced by localised heating from a thin resistance wire located in the middle; when the phase shift is removed by turning off the wire current, the laser is correspondingly turned off, as the threshold then exceeds the maximum gain available in the fibre. With the wire current optimally set, a maximum lasing output of $94 \mu \mathrm{W}$ (at $1570 \mathrm{~nm}$ ) was obtained for $167 \mathrm{~mW}$ pump power. A scanning Fabry-Perot interferometer confirmed single frequency laser operation.

Fig. 2 shows the green fluorescence observed by scanning along the laser (only the central $3 \mathrm{~cm}$ portion is shown, limited by the maximum travel of the translation stage available). The results, for 3 different pump/output powers, show that the fluorescence varies considerably over the length scanned, with a prominent peak in the centre where the phase shift is located.

Before deriving the relationship between the

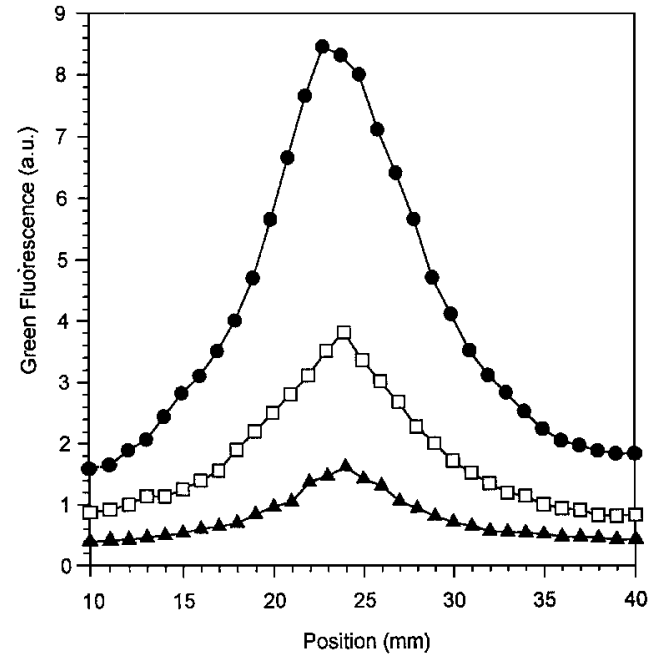

Fig. 2 Axial variation of green fluorescence intensity along laser, for different pump/ output powers:

$\triangle 74 \mathrm{~mW}$ pump, $46 \mu \mathrm{W}$ output power; $\square 110 \mathrm{~mW}$ pump, $66 \mu \mathrm{W}$ output power; $167 \mathrm{~mW}$ pump, $94 \mu \mathrm{W}$ output power. green fluorescence and the lasing intensity, we outline the physical basis for this connection. The green fluorescence arises from the excited state absorption of the 980nm pump [12], first exciting the erbium ions from the ${ }^{4} \mathrm{I}_{15 / 2}$ (ground) level to the ${ }^{4} \mathrm{I}_{11 / 2}$ level, and on to the ${ }^{4} \mathrm{~F}_{7 / 2}$ level (Fig. 3). After a fast decay to the ${ }^{4} \mathrm{~S}_{3 / 2}$ level, the ion relaxes to the ground state, yielding the green fluorescence. However, because of the fast nonradiative transition from the ${ }^{4} \mathrm{I}_{11 / 2}$ to the ${ }^{4} \mathrm{I}_{13 / 2}$ (metastable) level, most excited ions will accumulate in the long lifetime (10ms) metastable level, unavailable for excitation to the ${ }^{4} \mathrm{~S}_{3 / 2}$ level till they are de-excited back to the ground state. Hence, the presence of $1.55 \mu \mathrm{m}$ light, bringing the ions from the metastable back to the ground state via stimulated emission, results in increased green fluorescence. The rate equations describing the populations in the various levels (Fig. 3) are

$$
\begin{gathered}
d N_{4} / d t=\gamma_{3} N_{3}-N_{4} / T_{4} \\
d N_{3} / d t=\left(A_{23} N_{2}-A_{32} N_{3}\right) P_{p}-\gamma_{3} N_{3} \\
d N_{2} / d t=\left(A_{02} N_{0}-A_{20} N_{2}\right) P_{p}-\gamma_{2} N_{2}-\left(A_{23} N_{2}-A_{32} N_{3}\right) P_{p} \\
d N_{d} d t=\left(A_{20} N_{2}-A_{02} N_{0}\right) P_{p}+\left(A_{10} N_{1}-A_{01} N_{0}\right) P_{l}+N_{1} / T_{1}+N_{4} / N_{4}
\end{gathered}
$$

with $\mathrm{N}_{\mathrm{T}}=\mathrm{N}_{0}+\mathrm{N}_{1}+\mathrm{N}_{2}+\mathrm{N}_{3}+\mathrm{N}_{4}$ the total sum of ions. A ${ }_{\mathrm{ij}}$ are the absorption/emission coefficients from level $\mathrm{i}$ to level $\mathrm{j}$, i.e. $\mathrm{A}_{\mathrm{ij}}=/(\mathrm{h} \mathrm{A})$, with, $\mathrm{hv}$ and $\mathrm{A}$ the optical confinement 
factor, photon energy and fibre core area respectively, and ${ }_{\mathrm{ij}}$ the transition cross-section. $\mathrm{T}_{1}$ and $\mathrm{T}_{4}$ are the radiative lifetimes of levels 1 and 4 . In the analysis, we take $\mathrm{A}_{\mathrm{ij}}=\mathrm{A}_{\mathrm{ji}}$ with the exception of $\mathrm{A}_{10}$ and $\mathrm{A}_{01}$

Under steady state conditions, with the nonradiative rates ${ }_{3},{ }_{2}$ much greater than the other relevant transition rates, it can be shown that the green fluorescence $\mathrm{P}_{\mathrm{g}}$ varies as

$$
P_{g} \frac{P_{p}^{2}\left(A_{10} P_{l}+\frac{1}{T_{1}}\right)}{A_{20} P_{p}+\left(A_{10}+A_{01}\right) P_{l}+\frac{1}{T_{1}}}
$$

We note that in the absence of lasing $\left(\mathrm{P}_{1}=0\right)$, the green fluorescence has a background contribution $\mathrm{P}_{\mathrm{p}}{ }^{2} /\left(\mathrm{A}_{20} \mathrm{P}_{\mathrm{p}} \mathrm{T}_{1}+1\right)$. Subtracting this nonlasing contribution from (5), we have

$$
P_{g, \text { lasing }} \frac{P_{l} P_{p}^{2}}{A_{20} P_{p}+\left(A_{10}+A_{01}\right) P_{l}+\frac{1}{T_{1}}}\left\{A_{10}-\frac{A_{10}+A_{01}}{1+A_{20} P_{p} T_{1}}\right\}
$$

We see from eqn (6) that the lasing contribution to the green fluorescence $P_{g}$, lasing bears a simple relationship to $P_{l}$. In the case $P_{l} \ll P_{p}, P_{g}$, lasing is simply proportional to $P_{l}$. It should be mentioned that in this analysis, ion clustering has been neglected. In practice, most moderate/heavily doped $\mathrm{Er}^{3+}$-fibres have some of their ions clustered together, causing an unsaturable pump absorption, as intracluster energy transfer prevents more than one ion within each cluster remaining in the metastable state [13]. Thus, the majority of clustered ions are in the ground state, where they contribute a component to the background (nonlasing) green fluorescence. However, by subtracting the total measured background fluorescence, as described below, this complication can be ignored.

In the experiment, the background (nonlasing) contribution to the green fluorescence is measured by turning off the current to the resistance wire, and repeating the scan. Under these conditions, the green fluorescence is independent of position, as expected, due to the low pump absorption along the short laser length. Fig. 4 shows the intensity profile of

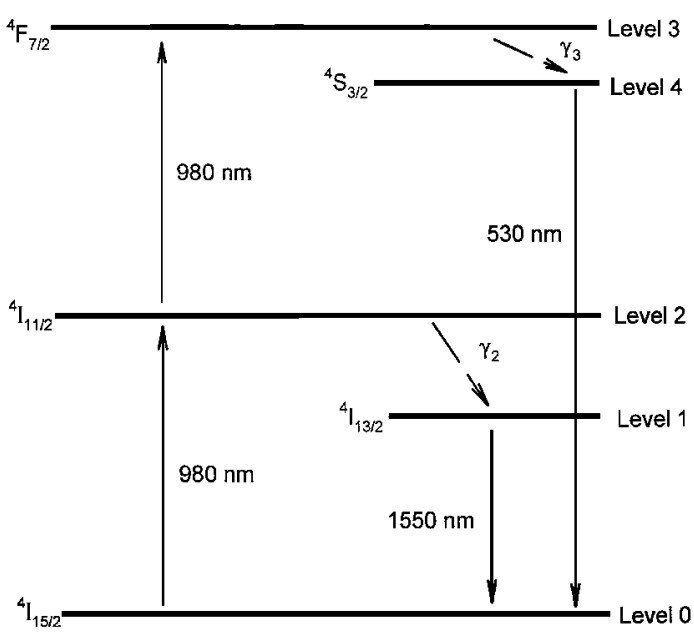

Fig. 3 Energy levels of the relevant transitions in erbium doped fibre. $P_{g, \text { lasing, }}$ obtained by subtracting this background portion from Fig. 2, for $74 \mathrm{~mW}$ pump power. The solid trace shows the theoretical intensity distribution (calculated by the transfer matrix approach) [14] for a $5 \mathrm{~cm}$ long discrete $\lambda / 4$ phase-shifted DFB laser with $\mathrm{L}=6.9$. The 'fit' to the experimental data is made simply by setting the theoretical peak equal to the experimental. We see that the green fluorescence data is indeed 
similar to the expected lasing profile, but slightly broader. This can be accounted for, because in a grating with such a large value of $\mathrm{L}$, the peak intensity in the centre of the laser should be $\sim 10^{3}$ times the output power. Therefore, even though the laser output is only $46 \mathrm{~mW}$, the intensity near the laser centre is actually comparable to the pump. $P_{g, \text { lasing }}$ is thus no longer simply proportional to the lasing intensity under these circumstances, due to the dependence on $P_{l}$ in the denominator of eqn (6). The dashed trace in Fig. 4 shows the expected profile of the green fluorescence calculated using eqn (6), in good agreement with the measured data. For this calculation, the theoretical distribution for $P_{l}$ was used, with $P_{p}=74 \mathrm{~mW}$, and the other parameters $A_{20}=10.8 \times 10^{4} \mathrm{~J}^{-1}, A_{10}$ $=10.3 \times 10^{4} \mathrm{~J}^{-1}=1.8 \mathrm{~A}_{01}, \quad T_{1}=$ $10 \mathrm{~ms}$.

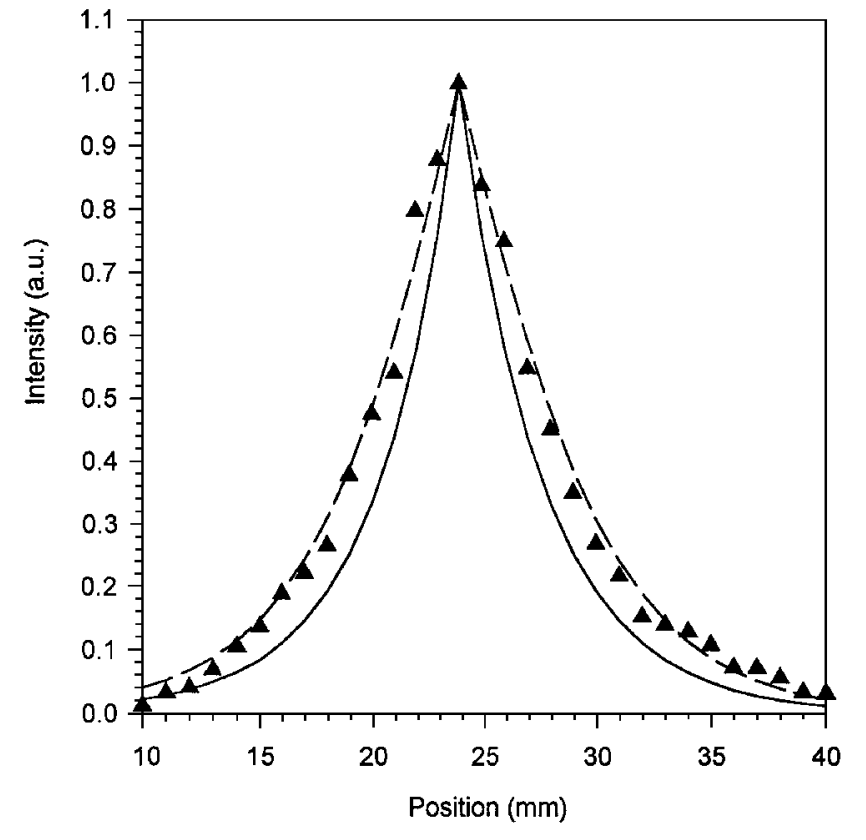

Fig. 4 Intensity distribution of $\mathbf{P}_{\text {g,lasing }}$ $(\Delta)$, the lasing contribution to the green fluorescence. Solid trace: Theoretical lasing profile for quarterwave phase-shifted DFB laser with grating strength $\mathbf{L}=$ 6.9. Dashed trace: Theoretical profile of green fluorescence from eqn (6).

The sharp intensity distribution in this laser clearly results in substantial spatial hole burning of the gain medium. However, as mentioned earlier, the accompanying refractive index variation in this moderately doped fibre should be negligible. This is consistent with the laser continuing to maintain stable single frequency operation in spite of the strong nonuniformity present in the intensity/gain profiles. Incorporating just the spatial gain variation, taking the gain dependence as $G \propto\left(A_{20} T_{1} P_{p}+\left(A_{10}+A_{01}\right) T_{1} P_{l}+1\right)^{-1}$, into the matrix calculations for the intensity distribution, we found no significant change to the expected profile. However, the fluorescence profiles obtained at the higher pump powers of $110 \mathrm{~mW}$ and $167 \mathrm{~mW}$ (Fig. 2) do show successive broadening of about $10 \%$ and $20 \%$ respectively. The cause of this broadening is unclear at this stage, but it may be due to heating effects in the centre of the laser at these higher powers. More detailed investigations are necessary to resolve this issue. In conclusion, we have demonstrated a simple but effective experimental technique for probing the intensity distribution in distributed feedback fibre lasers. By scanning along the laser length and monitoring the side green fluorescence, we have verified the distinctive peaked intensity distribution predicted for discrete phase-shifted DFB lasers. This technique should be very useful for the study and optimisation of more complex DFB structures.

\section{Acknowledgement}

This work was supported by the European Community ACTS project PHOTOS. The Optoelectronics Research Centre is an EPSRC-funded Interdisciplinary Research Centre. 


\section{References}

1. H.Soda, Y.Kotaki, H.Sudo, H.Ishikawa, S.Yamakoshi and H.Imai, IEEE J. Quantum Electron. QE-23, 804 (1987).

2. E.A.Whiteaway, G.H.B.Thompson, A.J.Collar and C.J.Armistead, IEEE J. Quantum Electron. QE-25, 1261 (1989).

3. P.Zhou and G.S.Lee, Electron. Lett., 26, 1660 (1990).

4. H.A.Haus and C.V.Shank, IEEE J. Quantum Electron. QE-12, 532 (1976).

5. L.J.P.Ketelsen, I.Hoshino and D.A.Ackerman, IEEE J. Quantum Electron. QE-27, 957 (1991).

6. M.R.Phillips, T.E.Darcie and E.J.Flynn, IEEE Photon. Technol. Lett. 4, 1201 (1992).

7. W.H.Loh and R.I.Laming, Electron. Lett. 31, 1440 (1995).

8. M.Sejka, P.Varming, J.Hubner and M.Kristensen, Electron. Lett. 31, 1445 (1995).

9. W.H.Loh, B.N.Samson, Z.E.Harutjunian and R.I.Laming, Electron. Lett. 32, 1204 (1996).

10. S.J.Frisken, Opt. Lett. 17, 1776 (1992).

11. J.Canning, M.Janos and M.G.Sceats, Opt. Lett. 21, 609 (1996).

12. E. Desurvire, Erbium Doped Fiber Amplifiers, (Wiley-Interscience, New York 1994) Chap. 4

13. R.S.Quimby, W.J.Miniscalco and B.Thompson, J. Appl. Phys. 76, 4472 (1994).

14. G.Bjork and O.Nilsson, J. Lightwave Technol. LT-5, 140 (1987). 